\title{
Survivability of Kluyveromyces marxianus Isolated From Korean Kefir in a Simulated Gastrointestinal Environment
}

OPEN ACCESS

Edited by:

Moshe Shemesh, Agricultural Research Organization

$(A R O)$, Israel

Reviewed by:

Graciela Liliana Garrote, National University of La Plata

Argentina

Yigal Achmon,

Guangdong Technion-Israel Institute

of Technology (GTIIT), China

*Correspondence:

Kun-Ho Seo

bracstu3@konkuk.ac.kr

${ }^{\dagger}$ These authors have contributed equally to this work

Specialty section: This article was submitted to

Food Microbiology,

a section of the journal

Frontiers in Microbiology

Received: 23 December 2021

Accepted: 25 January 2022

Published: 24 February 2022

Citation:

Youn $H-Y$, Kim D-H, Kim H-J, Bae D, Song K-Y, Kim H and Seo K-H (2022) Survivability of Kluyveromyces marxianus Isolated From Korean Kefir in a Simulated Gastrointestinal
Hye-Young Youn ${ }^{1+}$, Dong-Hyeon Kim ${ }^{1 \dagger}$, Hyeon-Jin Kim ${ }^{1}$, Dongryeoul Bae', Kwang-Young Song ${ }^{1}$, Hyunsook Kim ${ }^{2}$ and Kun-Ho Seo ${ }^{1 *}$

${ }^{1}$ Center for One Health, College of Veterinary Medicine, Konkuk University, Seoul, South Korea, ${ }^{2}$ Department of Food \& Nutrition, College of Human Ecology, Hanyang University, Seoul, South Korea

Kluyveromyces marxianus accounts for $>90 \%$ of the yeast population of kefir, and recently, its probiotic potential has been actively explored with a focus on its health benefits and safety. Herein, the survivability of five kefir-isolated $K$. marxianus strains $(\mathrm{Km}$ A1-A5) in a simulated gastrointestinal (GI) environment was evaluated and compared with those of commercial probiotic yeast, Saccharomyces boulardii MYA-796. To further explore the potential to survive in the host Gl tract, biochemical activities, hydrophobicity assay, biofilm formation, auto-aggregation analysis, and phenol tolerance of the strains were assessed. K. marxianus A4 exhibited the best survivability among all tested strains, including the clinically proven probiotic yeast strain S. boulardii MYA-796 $(p=0.014)$ in the artificial $\mathrm{Gl}$ tract ranging from $\mathrm{pH} 2.0$ to 7.5. In addition, the five $K$. marxianus strains and S. boulardii MYA-796 displayed different assimilation of lactose, xylitol, D-sorbitol, and DL-lactate, indicating that $K$. marxianus metabolized a wide range of substances and, thus, might be more feasible to nourish themselves in the host Gl tract for survival. K. marxianus strains showed a greater hydrophobicity of cell surface, abilities to biofilm formation and auto-aggregation, and phenol tolerance than S. boulardii MYA-796, suggesting greater potential for survival in the host Gl tract.

Keywords: kefir yeast, Kluyveromyces marxianus, probiotics, survivability, gastrointestinal environment

\section{INTRODUCTION}

The term "probiotic" means "for life" and refers to live microorganisms providing health benefits to the host in adequate amounts (Quigley, 2019). The basic requirements for good probiotics include survivability in the host gastrointestinal (GI) tract, beneficial health effects, and non-pathogenicity (Kim et al., 2019b).

To date, lactic acid bacteria have been the most prominent and renowned probiotic microorganisms. However, recently, yeasts have received increasing attention as promising probiotics, mainly due to their better resistance to various environmental stresses, lower possibility of acquisition and transfer/distribution of antibiotic resistance, and differential immune signaling to the host when compared to lactic acid bacterial probiotics (Tambekar and Bhutada, 2010). In addition to these advantages, many studies over the past decades have explored the potential of 
novel yeast species as probiotic microorganisms (Kelesidis and Pothoulakis, 2012). For instance, Saccharomyces boulardii is widely accepted as a probiotic yeast that has been proven prevention of acute diarrhea in double-blind experiments (Sazawal et al., 2006; Czerucka et al., 2007; Organic Materials Review Institute [OMRI], 2014).

Kefir is a traditional dairy product containing multiple probiotic microorganisms, primarily lactic acid bacteria, such as Lactobacillus kefiranofaciens and Lactobacillus kefiri, and yeasts including Kluyveromyces marxianus and Saccharomyces unisporus (Kim et al., 2019a). Among these, K. marxianus has been reported as the major yeast population in kefir (Kim et al., 2015, 2020). Several studies have investigated the probiotic attributes of $K$. marxianus, including its adhesion to the intestinal epithelium, antagonism toward pathogenic bacteria, anti-microbial, and anti-inflammatory functions (Lane and Morrissey, 2010; Liu et al., 2012; Romanin et al., 2016). Our previous study also evaluated the safety of $K$. marxianus by characterizing its phenotypic traits associated with virulence, such as the formation of pseudo-hyphae, production of proteinases, and hemolytic activity (Lim et al., 2019; Youn et al., 2022).

Probiotic strains to be applied to hosts evaluate not only particular functional attributes but also typical attributes under extreme conditions such as survival and maintenance in the artificial GI tract and phenolic environments (Bao et al., 2010). There are various ways to simulate the survivability of potential probiotic agents in GI environment, including acid and bile tolerances, mimicking physical conditions of GI tract, and persistence during gut transit in animal models (Diosma et al., 2014; Cudennec et al., 2015; Kim et al., 2017). In addition, adhesion to intestinal cells may also reflect the same properties because probiotics can survive in the host. The hydrophobicity of a cell may depend on the expression of various surfaceassociated proteins between strains and change with variation in the physiological state of cells and strains of species (Nwanyanwu et al., 2012). A high percentage of biofilm formation and autoaggregation of cells contribute to survival in the host GI tract and confer a competitive advantage over enteric bacterial pathogens (Suvarna et al., 2018). Furthermore, the auto-aggregation provides a protective barrier when probiotic strains form biofilms in the host GI tract and exopolysaccharides produced during biofilm formation inhibit pathogenic bacteria (Dertli et al., 2015). As these properties determined by the hydrophobicity of cell surface contribute to the activation of probiotics in humans, they are used to screen potential probiotics (Pan'kova et al., 2011). Relatively few studies have been conducted focusing on the survivability and the factors involved in the survival of the $K$. marxianus strains compared to the studies on the same aspects of S. boulardii.

In this study, we aimed to evaluate the survivability of five K. marxianus strains isolated from kefir in a simulated GI environment mimicking various physical conditions. Moreover, biochemical characteristics, hydrophobicity, biofilm formation, auto-aggregation, and phenol tolerance, that might affect their survivability in the GI environment, were also analyzed to further explore any correlations among them.

\section{MATERIALS AND METHODS}

\section{Experimental Design}

A schematic flowchart of the experimental procedures used to screen potential probiotic yeast agents isolated from Korean kefir is shown in Figure 1.

\section{Isolation of Yeast Strains From Kefir}

As a fermentation starter, kefir grain was obtained from the Center for One Health, Konkuk University, South Korea. Kefir was prepared by adding $50 \mathrm{~g}$ of viable kefir grains to $1 \mathrm{~L}$ of sterilized milk (Seoul Milk, Seoul Milk Cooperative, Seoul, South Korea) and fermenting the mixture at $25^{\circ} \mathrm{C}$ for $24 \mathrm{~h}$. Next, the grains and milk were separated using a sterilized plastic filter ( $2 \mathrm{~mm}$ pore size). Kefir milk was prepared daily during the experimental period. To isolate $K$. marxianus strains from kefir, we streaked kefir milk on potato dextrose agar (PDA; Oxoid, Basingstoke, Hampshire, United Kingdom) and incubated aerobically at $30^{\circ} \mathrm{C}$ for $72 \mathrm{~h}$. S. boulardii ATCC MYA-796 (Sb MYA-796) strain was kindly proved by prof. Dr. Hong-Gu Lee, College of Animal Bioscience \& Technology, Konkuk University (Seoul, South Korea). Sb MYA-796 was used as a control strain since it is the most studied and only clinically demonstrated probiotic yeast strain (Sazawal et al., 2006). Strains were grown on PDA at $30^{\circ} \mathrm{C}$ for $24 \mathrm{~h}$, and the cells were then sub-cultured twice under aerobic conditions.

\section{DNA Extraction}

Genomic DNA was extracted using the NucliSENS easyMAG instrument (bioMérieux, Marcy l'Etoile, France) in accordance with the manufacturer's instructions. Briefly, each colony was lysed in $1 \mathrm{~mL}$ of lysis buffer, and the lysate was incubated at $25^{\circ} \mathrm{C}$ for $30 \mathrm{~min}$. The lysed sample was then transferred to a plastic vessel containing $50 \mu \mathrm{L}$ of magnetic silica and subjected to automatic magnetic bead separation. The extracted DNA was resuspended in $25 \mu \mathrm{L}$ of elution buffer.

\section{Identification of Yeast Strains}

Five K. marxianus strains were identified via sequencing of the internal transcribed spacer (ITS) region (Diosma et al., 2014). Briefly, the primer pair sequences for ITS sequencing are as follows: ITS1: 5' - TCC GTA GGT GAA CCT GCG G-3' and ITS4: 5' - TCC TCC GCT TAT TGA TAT GC-3'. Polymerase chain reaction $(\mathrm{PCR})$ products were sequenced using the same primers and the ABI BigDye Terminator v3.1 Cycle Sequencing Kit (Applied Biosystems Carlsbad, CA, United States) according to the manufacturer's instructions. Sequencing was performed with the initial denaturation at $94^{\circ} \mathrm{C}$ for $5 \mathrm{~min}, 35$ cycles of denaturation at $94^{\circ} \mathrm{C}$ for $1 \mathrm{~min}$, annealing at $56^{\circ} \mathrm{C}$ for $1 \mathrm{~min}$, and extension at $72^{\circ} \mathrm{C}$ for $2 \mathrm{~min}$ followed by a final extension at $72^{\circ} \mathrm{C}$ for $10 \mathrm{~min}$ using an Applied Biosystems 3730XL DNA Analyzer by Bionics Co., Ltd (Seoul, South Korea). Obtained ITS sequences were aligned for each strain and were subjected to BLAST $^{1}$ using the NCBI rRNA/ITS database for identification at

\footnotetext{
${ }^{1}$ https://blast.ncbi.nlm.nih.gov/Blast.cgi
} 


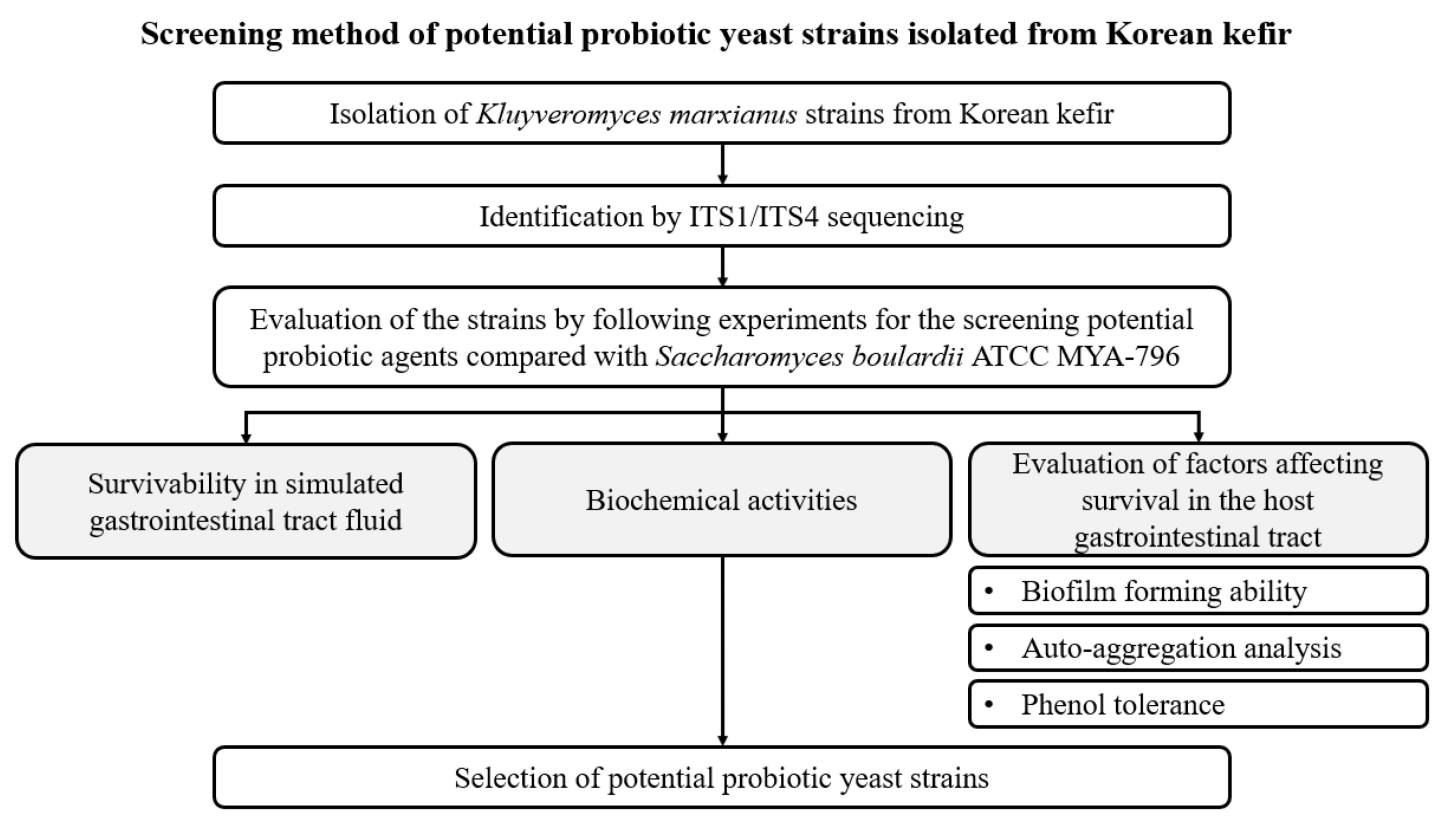

FIGURE 1 | Flowchart of the experimental procedures in the present study.

the species level. The identities of the isolates were determined on the highest BLAST score.

\section{Evaluation of Survivability/Growth Potential in Simulated Gastrointestinal Tract Conditions}

Survival of the yeast strains in the simulated GI tract was evaluated according to previous reports with some modifications (Cudennec et al., 2015; Ceugniez et al., 2017). The composition of the simulated fluids is given in Supplementary Table 1. Briefly, five K. marxianus and Sb MYA-796 were each suspended in potato dextrose broth (PDB; Difco, Detroit, MI, United States) and adjusted to $2.5 \mathrm{McF}$ arland [approximately $10^{5}$ colony forming units $(\mathrm{CFU}) / \mathrm{mL}]$. The yeast strains prepared at $10^{5} \mathrm{CFU} / \mathrm{mL}$ were added to the gastric fluid adjusted to $\mathrm{pH}$ 2.0 and 3.0, respectively. The gastric and intestinal fluids were incubated aerobically for $2 \mathrm{~h}$ or anaerobically for $24 \mathrm{~h}$, respectively, with agitation at $80 \mathrm{rpm}$ and $37^{\circ} \mathrm{C}$. The gastric fluid containing yeasts were then diluted at a $1: 1$ ratio using the intestinal fluid adjusted to $\mathrm{pH} 7.5$ and 8.5 (simulated serial GI environment I, pH 2.0 for gastric and 7.5 for the intestinal environment; simulated serial GI environment II, pH 2.0 for gastric and 8.5 for the intestinal environment; simulated serial GI environment III, pH 3.0 for gastric and 7.5 for the intestinal environment, and GI environment IV; $\mathrm{pH} 3.0$ for gastric and 8.5 for the intestinal environment) to provide the test strains with sequential exposure to gastric and intestinal environments. The samples were serially diluted with sterile phosphate-buffered saline (PBS; Sigma-Aldrich, St. Louis, MO, United States) before (for enumeration of initially viable cells) and after treatment (for enumeration of final viable cells), and viable colonies were enumerated on PDA (Oxoid) for $24 \mathrm{~h}$ at $37^{\circ} \mathrm{C}$.

\section{Biochemical Analysis of Yeast Strains}

The biochemical analysis of the yeast species was conducted using the $\operatorname{VITEK}^{\circledR} 2$ system (bioMérieux) in accordance with the manufacturer's instructions. Briefly, colonies of each yeast strain were suspended in $0.45 \%$ saline and allowed to approach McFarland 2.0 turbidity. Then, the suspension was placed in the VITEK $^{\circledR} 2$ system and analyzed using the YST card (bioMérieux). YST cards were incubated at $35.5^{\circ} \mathrm{C}$ for $18 \mathrm{~h}$, with optical readings automatically obtained every $15 \mathrm{~min}$.

\section{Evaluation of Factors Affecting Survival in the Host Gastrointestinal Tract Hydrophobicity Assay}

The hydrophobicity of the five K. marxianus strains and $\mathrm{Sb}$ MYA-796 was evaluated using the microbial adhesion to solvent (MATS) method described by Bellon-Fontaine et al. (1996). The hydrophobic solvents used were hexadecane (analytical standard grade, 99.8\%, Sigma-Aldrich, St. Louis, MO, United States), decane (anhydrous grade, 99\%, Sigma-Aldrich), and chloroform (anhydrous grade, 99\%, Sigma-Aldrich). In brief, the yeast strains were cultivated in $\mathrm{PDB}$ (Difco) at $37^{\circ} \mathrm{C}$ for $24 \mathrm{~h}$ and centrifuged at $7,280 \times g$ for $10 \mathrm{~min}$. The resulting pellet was washed twice with sterile PBS (Sigma-Aldrich) and resuspended in PBS at approximately $10^{5} \mathrm{CFU} / \mathrm{mL}$. The optical density (OD) of the suspension was measured (A0) at a wavelength of $405 \mathrm{~nm}$ using a microplate reader (Multiskan FC, Thermo Fisher Scientific, Shanghai, China). Next, $280 \mu \mathrm{L}$ of each yeast suspension was vortexed for $90 \mathrm{~s}$ with $40 \mu \mathrm{L}$ of each solvent. The mixture was allowed to stand for 15 min to ensure complete separation of the two phases. The OD of the water phase was then measured (A1) at a wavelength of $405 \mathrm{~nm}$. The percentage of cell surface hydrophobicity was calculated using the following 
equation:

$$
\text { Hydrophobicity }(\%)=(1-\mathrm{A} 1 / \mathrm{A} 0) \times 100 \%
$$

The percentage of hydrophobicity was expressed as follows: $0-$ $35 \%$, low hydrophobicity; 36-70\%, medium hydrophobicity; and 71-100\%, high hydrophobicity (Mladenović et al., 2020).

\section{Biofilm Formation}

The biofilm-forming ability of the yeast strains was evaluated as previously described (Jeong et al., 2018). In brief, each colony of the isolates was added to $200 \mu \mathrm{L}$ PDB (Difco) and set to 3.0 McFarland turbidity. To evaluate the biofilm formation, $200 \mu \mathrm{L}$ of each sample was transferred to a 96-well polystyrene culture plate (SPL Life Sciences, Gyeonggi-do, South Korea) and incubated at $37^{\circ} \mathrm{C}$ for $24 \mathrm{~h}$. The culture medium was discarded, and the microplate was gently washed twice with $200 \mu \mathrm{L}$ PBS (Sigma-Aldrich). Cells were stained with $0.1 \%(\mathrm{w} / \mathrm{v})$ crystal violet (100 $\mu \mathrm{L}$; Sigma-Aldrich, St. Louis, MO, United States) for $15 \mathrm{~min}$ at room temperature $\left(20-25^{\circ} \mathrm{C}\right)$ and rinsed twice with $\mathrm{PBS}$ (Sigma-Aldrich). After removing the dye with $200 \mu \mathrm{L}$ of $99 \%$ ethanol, the biofilm was quantified by measuring the absorbance at $595 \mathrm{~nm}$ using a Multiskan FC (Thermo Fisher Scientific).

\section{Auto-Aggregation Assay}

Auto-aggregation assay was performed according to the method of Fonseca et al. (2021) with slight modifications. Briefly, yeast strains were pelleted in PBS (Sigma-Aldrich) and adjusted to obtain $10^{5} \mathrm{CFU} / \mathrm{mL}$ in the same buffer. The OD of the suspension before (A0) and after $5 \mathrm{~h}$ incubation at $37^{\circ} \mathrm{C}(\mathrm{At})$ were measured at a wavelength of $595 \mathrm{~nm}$ using a Multiskan FC (Thermo Fisher Scientific). The incubation time of At was set in consideration of the lag phases of $K$. marxianus and S. boulardii to prevent the mistaken result of auto-aggregation due to the growth of the yeast strains. The plate was shaken for $5 \mathrm{sec}$ immediately before each reading. The auto-aggregation percentage was determined using the following equation:

$$
\text { Auto-aggregation }(\%)=(1-\mathrm{At} / \mathrm{A} 0) \times 100 \%
$$

\section{Phenol Tolerance}

Phenol tolerance of yeast strains was evaluated as described by Shehata et al. (2016) with slight modifications. Overnight cultures of yeast strains were inoculated (1\%) in PDB (Difco) with 0.2 and $0.5 \% \mathrm{v} / \mathrm{v}$ of phenol or without phenol. Yeast cells in the PDB were quantified by reading the $\mathrm{OD}_{620}$ after $24 \mathrm{~h}$ incubation at $37^{\circ} \mathrm{C}$.

\section{Statistical Analyses}

All experiments were performed in triplicate. SPSS version 25.0 (SPSS Inc., Chicago, IL) was used for data analysis. The final yeast cell count was divided by the initial cell count to express the survivability/growth potential and phenol tolerance (fold change); in Figures 2, 3, values below 1.0 indicated survivability, and those over 1.0 indicated fold-growth. All data were analyzed for normal distribution, and homogeneity of variance was conducted using one-way analysis of variance (ANOVA), followed by Duncan's post hoc analysis. Differences were considered significant at $p<0.05$.

\section{RESULTS}

\section{Yeast Strain Identification}

Intezrnal transcribed spacer (ITS) sequencing identified K. marxianus at the species level and their sequences (Accession number of MT791345 for K. marxianus A1; MT793595 for K. marxianus A2; MT793593 for K. marxianus A3; MT793596 for K. marxianus A4; and MT793594 for K. marxianus A5) were submitted to GenBank.

\section{Survival in Artificial Gastrointestinal Tract Fluid}

Five K. marxianus strains (Km A1-A5) and Sb MYA-796 were evaluated for their survivability in the simulated GI environment. K. marxianus strains grew in number ranging from 1.15 to 11.23 fold in various GI environments (Figure 2). In general, Km A4 and A5 showed the highest survivability among $\mathrm{Km}$ strains tested. In contrast, Sb MYA-796 did not show growth but only survived, ranging from 0.45 to 0.76 -fold in various environments. Interestingly, Km A4 showed significantly higher survivability than Sb MYA-796 in all simulated serial GI environments $(p<0.05)$. All yeast strains showed higher survivability or foldgrowth in the environment with higher $\mathrm{pH}$ scales (i.e., from the lowest $\mathrm{pH}$ scale of GI environment I to the highest of GI environment IV).

\section{Biochemical Activities}

Supplementary Table 2 represents the full list of the 46 biochemical characteristics of $\mathrm{Km} \mathrm{A1-A5}$ isolated from kefir as well as those of Sb MYA-796, evaluated using the VITEK ${ }^{\circledR} 2$ System. Overall, compared to Sb MYA-796, all K. marxianus strains displayed a broader range of biochemical activities: the number of positive/total tests (\%) were 16/46 (34.78\%), 17/46 (36.96\%), 14/46 (30.43\%), 16/46 (34.78\%), 18/46 (39.13\%), and 11/46 (23.91\%) for Km A1-A5 and Sb MYA-796, respectively. Selected biochemical tests are represented in Table 1 . The biochemical activities present in all K. marxianus strains and absent in Sb MYA-796 included lactose assimilation, xylitol assimilation, D-sorbitol assimilation, and DL-lactate assimilation (Table 1). Conversely, biochemical activities absent in all K. marxianus strains and present in Sb MYA-796 included D-maltose assimilation, D-turanose assimilation, and D-trehalose assimilation (Table 1).

Differing biochemical activities at the strain level were as follows: L-malate assimilation, amygdaline assimilation, $\mathrm{L}$-arabinose assimilation, $\mathrm{D}$-xylose assimilation, urease being positive only in $\mathrm{Km} \mathrm{A4}$, L-glutamate assimilation, L-proline assimilation, and tyrosine-arylamidase (Table $\mathbf{1}$ ).

\section{Evaluation of Factors Affecting Survival in the Host Gastrointestinal Tract Hydrophobicity Analysis}

The hydrophobicity of the cell surface of tested yeasts was evaluated using three hydrophobic solvents, including hexadecane, decane, and chloroform (Table 2). All K. marxianus strains had higher adhesion to hydrophobic solvents than Sb MYA-796, which exhibited medium hydrophobicity to all 

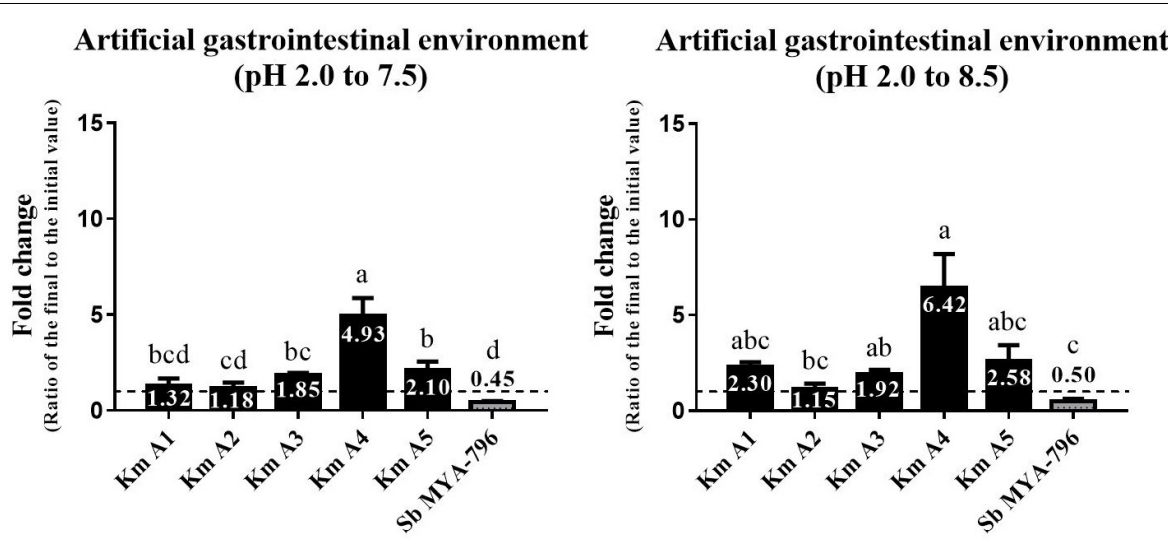

Artificial gastrointestinal environment (pH 3.0 to 7.5 )

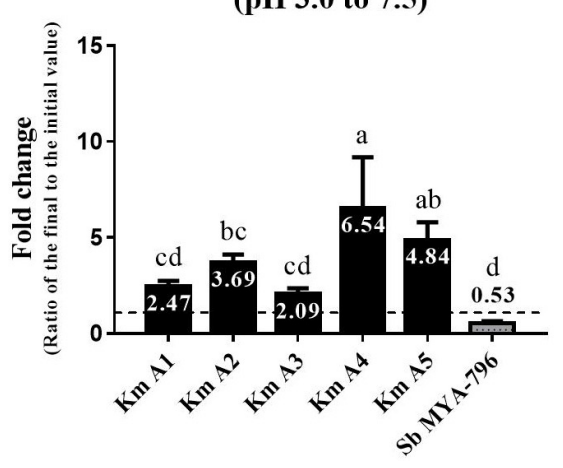

Artificial gastrointestinal environment (pH 3.0 to 8.5 )

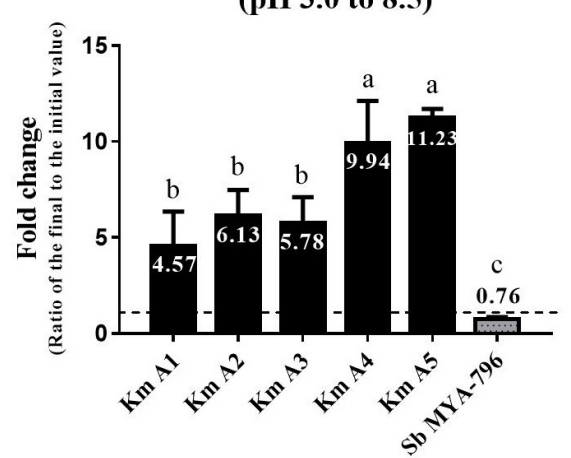

FIGURE 2 | Changes in the number of viable cells of the five Kluyveromyces marxianus strains isolated from kefir and Saccharomyces boulardii MYA-796 at pH 2.0 to $7.5,2.0$ to 8.5, 3.0 to 7.5 , and 3.0 to 8.5. Different letters above the bars indicate significant differences at $p<0.05$ using the Duncan method. The yeast strains were exposed to the simulated gastric fluid adjusted to $\mathrm{pH} 2.0$ and 3.0 and incubated with agitation for $2 \mathrm{~h}$ at $37^{\circ} \mathrm{C}$, respectively. Then, the yeast-containing gastric fluids were diluted using intestinal fluids adjusted to $\mathrm{pH} 7.5$ and 8.5 to provide sequential exposure to gastric and intestinal environments and incubated with agitation for $24 \mathrm{~h}$ at $37^{\circ} \mathrm{C}$, respectively. All experiments were performed in triplicate. $\mathbf{K m} \mathbf{A 1}, K$. marxianus A1; $\mathbf{K m} \mathbf{A 2}, K$. marxianus A2; $\mathbf{K m} \mathbf{A} 3, K$. marxianus A3; Km A4, K. marxianus A4; Km A5, K. marxianus A5; Sb MYA-796, S. boulardii MYA-796.
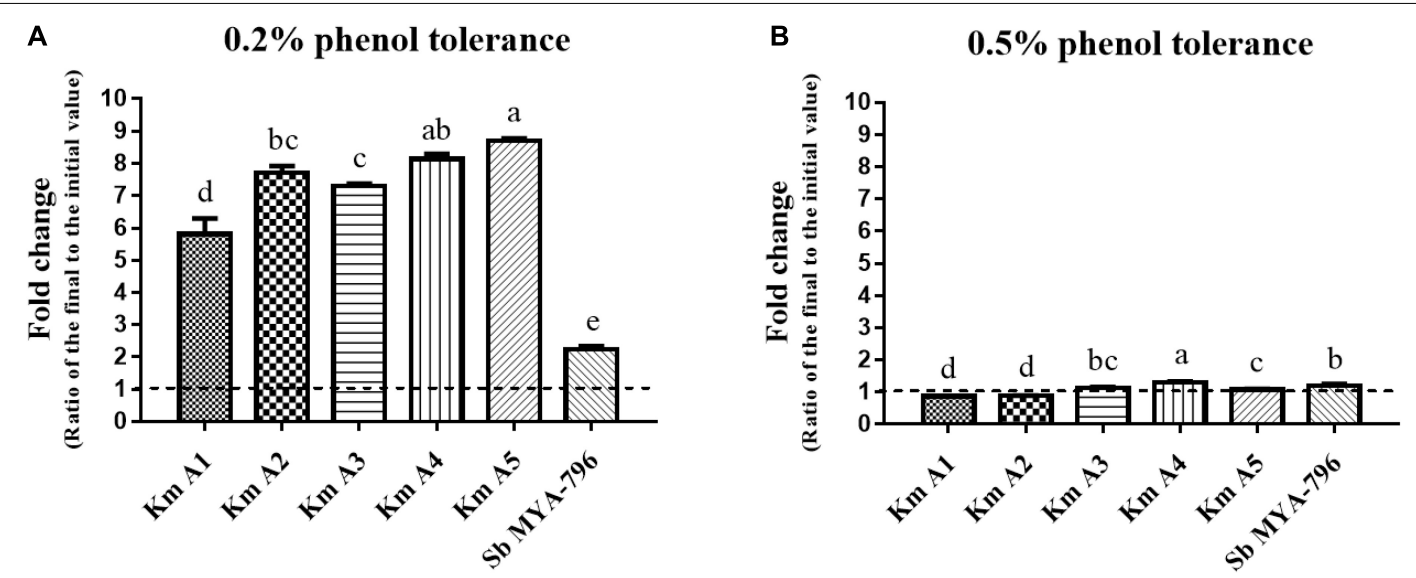

FIGURE 3 | Effect of phenol concentration on the growth of the Kluyveromyces marxianus strains and Saccharomyces boulardii MYA-796. (A) The 0.2\% phenol tolerance and (B) $0.5 \%$ phenol tolerance of all yeast strains. Different letters above the bars at the same phenol tolerance indicate significant differences at $p<0.05$ using the Duncan method. All experiments were performed in triplicate. Km A1, K. marxianus A1; Km A2, K. marxianus A2; Km A3, K. marxianus A3; Km A4, K. marxianus A4; Km A5, K. marxianus A5; Sb MYA-796, S. boulardii MYA-796. 
TABLE 1 | Selected biochemical activities of the five Kluyveromyces marxianus strains isolated from kefir and Saccharomyces boulardii MYA-796 differing at the species or strain levels, as analyzed using the VITEK ${ }^{\circledR} 2$ system.

\begin{tabular}{|c|c|c|c|c|c|c|c|}
\hline \multirow[t]{2}{*}{ Associated metabolism } & \multirow[t]{2}{*}{ Biochemical test } & \multicolumn{6}{|c|}{ Yeast strain } \\
\hline & & Km A1 & $\mathrm{Km} \mathrm{A2}$ & $\mathrm{Km} \mathrm{A3}$ & Km A4 & Km A5 & Sb MYA-796 \\
\hline \multirow[t]{11}{*}{ Carbohydrate metabolism } & Lactose assimilation $(\mathrm{LACa})^{\dagger}$ & + & + & + & + & + & - \\
\hline & Xylitol assimilation $(\mathrm{XLTa})^{\dagger}$ & + & + & + & + & + & - \\
\hline & D-sorbitol assimilation (dSORa) ${ }^{\dagger}$ & + & + & + & + & + & - \\
\hline & DL-lactate assimilation $\left(\llcorner A T a)^{\dagger}\right.$ & + & + & + & + & + & - \\
\hline & D-maltose assimilation (dMALa) ${ }^{\dagger}$ & - & - & - & - & - & + \\
\hline & D-turanose assimilation (dTURa) ${ }^{\dagger}$ & - & - & - & - & - & + \\
\hline & D-trehalose assimilation (dTREa) ${ }^{\dagger}$ & - & - & - & - & - & + \\
\hline & L-malate assimilation $(\mathrm{IMLTa})^{\ddagger}$ & + & + & - & - & + & - \\
\hline & Amygdaline assimilation (AMYa) $)^{\ddagger}$ & + & + & - & + & + & + \\
\hline & L-arabinose assimilation (IARAa) $)^{\ddagger}$ & - & - & + & + & + & - \\
\hline & D-xylose assimilation (dXYLa) $)^{\ddagger}$ & - & + & + & + & + & - \\
\hline \multirow[t]{4}{*}{ Protein metabolism } & Urease $(\mathrm{URE})^{\ddagger}$ & - & - & - & + & - & - \\
\hline & L-glutamate assimilation $(\mathrm{IGLTa})^{\ddagger}$ & + & + & + & - & + & - \\
\hline & L-proline assimilation $(\mathrm{IPROa})^{\ddagger}$ & + & + & - & - & + & - \\
\hline & Tyrosine-arylamidase $(\text { TyrA })^{\ddagger}$ & + & + & - & + & + & - \\
\hline
\end{tabular}

${ }^{\dagger}$ Biochemical activities of five K. marxianus strains and S. boulardii MYA-796 differing at the species level.

${ }^{\ddagger}$ Biochemical activities of five K. marxianus strains and S. boulardii MYA-796 differing at the strain level.

Km A1, K. marxianus A1; Km A2, K. marxianus A2; Km A3, K. marxianus A3; Km A4, K. marxianus A4; Km A5, K. marxianus A5; Sb MYA-796, S. boulardii MYA-796.

TABLE 2 | Adhesion properties of Kluyveromyces marxianus strains isolated from kefir compared with that of Saccharomyces boulardii MYA-796.

\begin{tabular}{lccc}
\hline Yeast strains & \multicolumn{3}{c}{ Hydrophobicity (\%) } \\
\cline { 2 - 4 } & Hexadecane & Decane & Chloroform \\
\hline Km A1 & $66.95 \pm 3.41^{\mathrm{c}}$ & $71.41 \pm 1.37^{\mathrm{b}}$ & $69.05 \pm 0.88^{\mathrm{d}}$ \\
$\mathrm{Km} \mathrm{A2}$ & $72.04 \pm 1.24^{\mathrm{b}}$ & $71.67 \pm 0.62^{\mathrm{b}}$ & $73.91 \pm 4.35^{\mathrm{c}}$ \\
$\mathrm{Km} \mathrm{A3}$ & $67.89 \pm 2.71^{\mathrm{c}}$ & $60.93 \pm 1.11^{\mathrm{c}}$ & $65.38 \pm 3.55^{\mathrm{e}}$ \\
Km A4 & $76.89 \pm 4.03^{\mathrm{a}}$ & $73.80 \pm 1.21^{\mathrm{a}}$ & $82.18 \pm 3.55^{\mathrm{a}}$ \\
$\mathrm{Km} \mathrm{A5}$ & $75.02 \pm 2.14^{\mathrm{ab}}$ & $70.11 \pm 2.07^{\mathrm{b}}$ & $77.88 \pm 2.50^{\mathrm{b}}$ \\
$\mathrm{Sb} \mathrm{MYA-796}$ & $59.44 \pm 1.81^{\mathrm{d}}$ & $60.28 \pm 0.67^{\mathrm{c}}$ & $59.36 \pm 1.99^{\mathrm{f}}$
\end{tabular}

Different letters in a column indicate significant differences $(p<0.05)$ using the Duncan method.

Km A1, K. marxianus A1; Km A2, K. marxianus A2; Km A3, K. marxianus A3; Km A4, K. marxianus A4; Km A5, K. marxianus A5; Sb MYA-796, S. boulardii MYA-796.

solvents. On the other hand, Km A2, A4, and A5 displayed high hydrophobicity with more than $70 \%$ adhesion. Interestingly, the most hydrophobic strain was $\mathrm{Km} \mathrm{A4}$, showing a significantly high affinity to hexadecane, decane, and chloroform $(p<0.05)$.

\section{Biofilm-Forming Ability}

The biofilm-forming ability of $\mathrm{Km}$ A1-A5 was significantly different from that of Sb MYA-796 after $24 \mathrm{~h}$ at $37^{\circ} \mathrm{C}(p<0.05$; Figure 4A). The mean $\mathrm{OD}_{595}$ value of $\mathrm{Km} \mathrm{A} 1-\mathrm{A} 5$ was observed to be $0.3960,0.4098,0.4226,0.4160$, and 0.3874 , respectively, whereas Sb MYA-796 showed a weaker capacity to form biofilms (mean $\mathrm{OD}_{595}$ value of 0.1118 ) than all K. marxianus strains.

\section{Auto-Aggregation Analysis}

The yeast strains evaluated in the present study had values of auto-aggregation ranging from 68.67 to $84.66 \%$ after $5 \mathrm{~h}$ of incubation (Figure 4B). The highest values were found for $\mathrm{Km} \mathrm{A5}$, which exhibited an auto-aggregation value of $84.66 \%$, whereas that of Sb MYA-796 was $68.67 \%(p<0.05)$.

\section{Phenol Tolerance}

The effect of different phenol concentrations (0.2 and $0.5 \%$ ) on the growth of Km A1-A5 and Sb MYA-796 was determined, as shown in Figure 3. All strains were more tolerant to $0.2 \%$ phenol than $0.5 \%$ phenol. In $0.2 \%$ phenol solution, Km A5 (8.70-fold) was the most tolerant, followed by $\mathrm{Km} \mathrm{A} 4$ (8.14-fold), whereas Sb MYA-796 (2.24-fold) had the lowest value compared to that of Km A1-A5 ( $p<0.05$, Figure 3A). In $0.5 \%$ phenol solution, $\mathrm{Km} \mathrm{A} 4$ was the most tolerant, exhibiting 1.07-fold survivability compared to the other yeast strains ( $p<0.05$, Figure 3B).

\section{DISCUSSION}

To the best of our knowledge, few studies have demonstrated the survivability and survival affecting factors of $K$. marxianus isolated from kefir in vitro with the most renowned probiotic yeast, $S$. boulardii, as a reference. The results highlighted greater survivability/growth potential of $K$. marxianus in the simulated serial GI environments and a broader range of biochemical activities compared to Sb MYA-796. In addition, the hydrophobicity of cell surface, auto-aggregation, biofilm formation, and phenol tolerance of the K. marxianus strains were superior to these of S. boulardii MYA-796.

A previous study reported that the survivability of $K$. marxianus isolated from kefir in artificial gastric and intestinal environments for $30 \mathrm{~min}$ and $6 \mathrm{~h}$, respectively, was superior to that of Saccharomyces cerevisiae KCTC 7004 (You et al., 2006). Moreover, K. marxianus S97, S101, and S106 

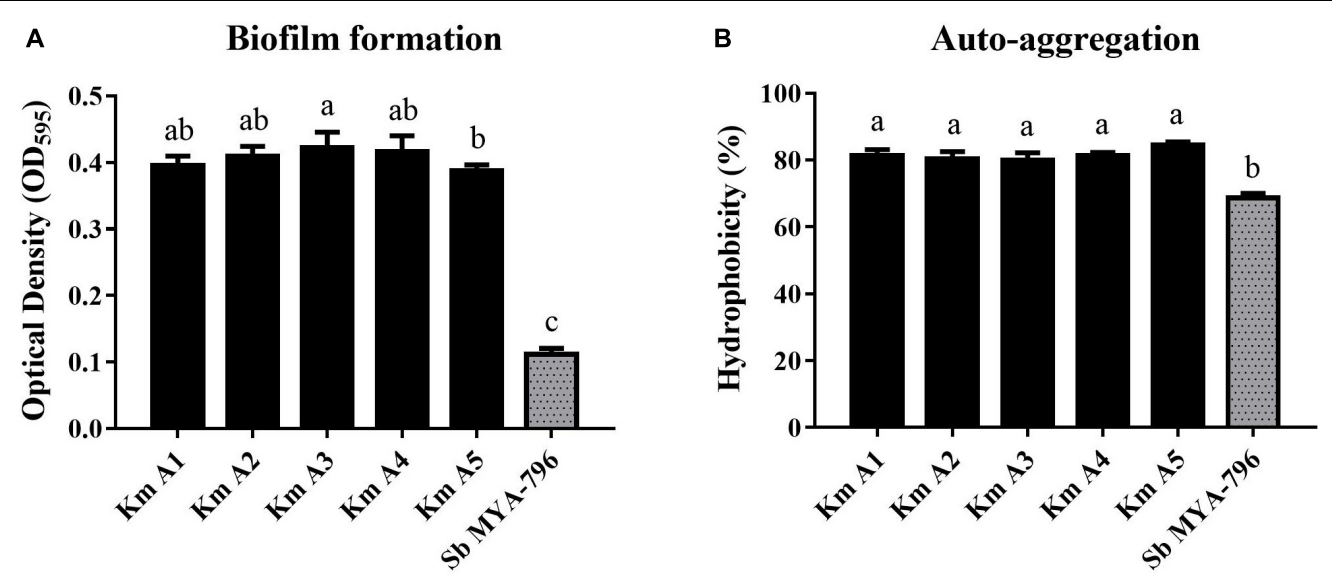

FIGURE 4 | (A) Biofilm formation and (B) auto-aggregation analysis of Kluyveromyces marxianus strains (Km A1-A5) compared with those of Saccharomyces boulardii MYA-796 at $37^{\circ} \mathrm{C}$. Error bars represent standard deviations. Different letters indicate statistical differences at $p<0.05$ using the Duncan method. All experiments were performed in triplicate. NC, Negative control (Potato dextrose broth); Km A1, K. marxianus A1; Km A2, K. marxianus A2; Km A3, K. marxianus A3; Km A4, K. marxianus A4; Km A5, K. marxianus A5; Sb MYA-796, S. boulardii MYA-796.

isolated from fruits and dairy samples survived approximately $\log 6.8 \mathrm{CFU} / \mathrm{mL}$ after exposure to $\mathrm{pH} 2.0$ for $96 \mathrm{~h}$ and showed higher survivability than S. cerevisiae S28 and S34 (approximately $\log 6.0 \mathrm{CFU} / \mathrm{mL}$ ) (Moradi et al., 2018). Another study suggested that, under simulated gastric conditions $(\mathrm{pH} 3.0)$ and duodenum juice, K. marxianus NS1KM2, 14KM1, and $6688 \mathrm{KM}$ isolated from Fiore Sardo cheese had better survival (decrease by $0-17 \%$ ) than S. boulardii CODEX SB1 (decrease by 64.5\%) (Fadda et al., 2017). Especially, in a recent study (Ceugniez et al., 2017), the survivability of $K$. marxianus S-2-05 decreased by 0.47 -fold in a consecutive simulated serial GI environment $(\mathrm{pH} 3.0$ to 7.0; $2 \mathrm{~h}$ for gastric condition and $2 \mathrm{~h}$ for intestinal condition), whereas in this study, Km A4 and Km A5 increased by 6.54 and 4.84-fold, respectively, in the same $\mathrm{pH}$ environment $(\mathrm{pH}$ 3.0 to $7.0 ; 2 \mathrm{~h}$ for gastric condition and $24 \mathrm{~h}$ for intestinal condition). The different survivability of yeast strains in the two studies is due to the different incubation times of simulated intestinal environments. Comparing viability for more than $12 \mathrm{~h}$ in an intestinal environment is important because it shows that microorganisms can not only survive but also grow in the environment. However, no previous studies have focused on the survivability or growth potential of $K$. marxianus versus $S$. boulardii over a wide range of $\mathrm{pH}$ in a consecutive simulated GI environment $(2 \mathrm{~h}$ for gastric condition and $24 \mathrm{~h}$ for intestinal condition). Here, we demonstrated that both $K$. marxianus and $S$. boulardii survived in the mimicking host GI tract; however, K. marxianus strains were more resistant than those of $S$. boulardii. The excellent survivability of $K$. marxianus evaluated in this study could benefit industries looking for yeast probiotics that can survive against preservatives other than Sb MYA-796.

Among kefir ecosystems, kefir yeasts appear to exert superior survivability to kefir lactic acid bacteria. Numerous survivability studies conducted on probiotic lactic acid bacteria have shown the relatively low survivability of these microorganisms; for example, Lactobacillus acidophilus M23 displayed a reduction of $4.1 \log \mathrm{CFU} / \mathrm{mL}$ in a gastric environment at $\mathrm{pH} 2.5$
(Song et al., 2015). In contrast, all K. marxianus strains displayed a reduction of less than $1 \log \mathrm{CFU} / \mathrm{mL}$ under highly acidic conditions. We previously reported that L. kefiranofaciens, Leuconostoc mesenteroides, and L. kefiri strains, isolated from kefir, not only survived but also grew to exceed the initial bacterial count in the gastric environment adjusted to $\mathrm{pH} 2.5$ with pepsin and intestinal environment adjusted to $\mathrm{pH} 7.0$ with $0.3 \%$ oxgall, respectively (Kim et al., 2017). However, Lactococcus lactis strains displayed $<50 \%$ survival in the gastric environment

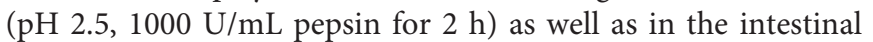
environment ( $\mathrm{pH}$ 8.0, 0.3\% oxgall for $24 \mathrm{~h}$ ) (Kim et al., 2017). In comparison with these historical data, the superior survivability of $K$. marxianus strains might partially support our recent findings that $\mathrm{Km}$ A5 exerted better competitive exclusion against Salmonella enterica serovar Enteritidis than L. kefiranofaciens DN1 in the GI tract of chicks (Bae et al., 2020).

Several studies have investigated the biochemical and metabolic aspects of different $K$. marxianus strains for potential application in a bioindustrial reactor rather than as probiotics (Fonseca et al., 2008). To date, many studies have explored the potential benefits of probiotic attributes of Lactobacillus and Bifidobacterium spp. on the host digestive system, such as enzymatic capacity, modulation of the metabolic functioning of the host, and alleviating the symptoms of several diseases (Rabot et al., 2010; Sánchez et al., 2017) using animal models and clinical interventions, neglecting the importance of the biochemical activities as a survival factor of the probiotic microorganism itself. Microorganisms that use a wide range of metabolic substrates are more likely to survive by decomposing the complex substrates into intermediate fermentation products, including fumarate, succinate, and lactate in the host GI tract and using them to obtain energy (Rowland et al., 2018). In this light, K. marxianus with a wide spectrum of biochemical activities might be helpful for the survivability of the host GI tract (Rabot et al., 2010).

K. marxianus constitutes the majority of lactose-utilizing yeasts in dairy products such as milk, and all K. marxianus 
strains tested were positive for lactose assimilation (Simova et al., 2002; Diosma et al., 2014). This ability could be attributed to two genes, lac4 and lac12, which encode a $\beta$-galactosidase and lactose permease, respectively (Lane and Morrissey, 2010). $\beta$-galactosidase from Kluyveromyces spp. and the filamentous fungus Aspergillus niger is the most common form of commercial lactase (Adam et al., 2005). Therefore, K. marxianus strains could alleviate lactose intolerance and more survive in the host GI tract than Saccharomyces spp. In different biochemical activities at the strain level, urease was positive only in $\mathrm{Km} \mathrm{A4}$, which showed the highest survivability in the most acidic gastric environment. Urease is considered a stress response that counteracts the effects of low $\mathrm{pH}$ environments and modulates intracellular and extracellular pH in some bacteria (Mora et al., 2004). Colonization of Helicobacter pylori in the stomach has been related to the presence of bacterial urease and urea metabolism (Ferrero et al., 1988). Probiotics such as Bifidobacterium longum subsp. infantis and Streptococcus thermophilus are urease-positive bacteria that colonize early in the GI tract of the host (Mora et al., 2004; LoCascio et al., 2010). Moreover, these urease-positive microorganisms share the environmental benefit of a temporal local $\mathrm{pH}$ increase with urease-negative microorganisms (Arioli et al., 2010). The urease-positive Km A4 consistently presented the highest survivability in the most acidic gastric environment.

Hydrophobic cell surface was demonstrated by high adherence to hexadecane, decane, and chloroform. Many studies have shown that the presence of glycoprotein on the cell surface results in higher hydrophobicity (Cuperus et al., 1993). In a previous study, when the hydrophobic ability was set as an affinity higher than $40 \%$ in hexadecane and chloroform, Lactobacillus paracasei lac 1, L. acidophilus lac 2 and 3, and Lactobacillus plantarum lac 6 showed hydrophobicity of the cell surface (Abdulla et al., 2014). According to another study, the affinity of $L$. acidophilus M92 and L. plantarum L4 for chloroform was $36.06 \%$ and $47.03 \%$, respectively (Kos et al., 2003). After comparison with a previous study, the biofilm formation ability of $K$. marxianus strains (Km A1-A5) isolated from kefir was superior to that of $K$. marxianus S-2-05 isolated from a traditional French cheese. Moreover, K. marxianus S-2-05 had a low affinity for hexadecane $(8.89 \%)$, decane $(20.13 \%)$, and chloroform (60.41\%) compared to the $\mathrm{Km} \mathrm{A1-A5,} \mathrm{implying} \mathrm{that} \mathrm{the}$ cell surface hydrophobicity and biofilm-forming ability depend on strain (Ceugniez et al., 2017). The relatively higher affinities to chloroform of kefir yeast strains indicate the basic character of the yeast cell, which is related to the presence of carboxylic groups on the microbial surface (BellonFontaine et al., 1996). Our results indicate that $K$. marxianus strains isolated from kefir have greater hydrophobicity, biofilm formation, and auto-aggregation properties than the probiotic Sb MYA-796.

Phenols are formed by bacterial degradation of the aromatic amino acids, inhibit bacteria in gut microbiota, and affect diversity and metabolic activity (Fonseca et al., 2021). Intestinal bacteria involved in phenolic formation include Bacteroides, Enterobacteriaceae, Lactobacillus, and Bifidobacterium (Hughes et al., 2000). In the gut environment, phenolic compounds may selectively inhibit or stimulate the growth of some of the intestinal microorganisms and can also affect bacteria population kinetics (Tzounis et al., 2008). Moreover, phenols have bacteriostatic effects against potential probiotic agents (Fonseca et al., 2021). Consistent results reported that a $0.4 \%$ phenol concentration causes a bacteriostatic action in L. acidophilus DC 602 and L. gasseri DC 422 (Xanthopoulos et al., 2000). Consequently, phenol tolerance is essential for the characterization of probiotic strains (Divisekera et al., 2019). To the best of our knowledge, this is the first study to demonstrate the phenol tolerance of potential probiotic yeast. $\mathrm{Km}$ A1-A5 showed a difference in sensitivity for different phenol concentrations but overall could tolerate the tested phenol concentrations. This could be noted given a recent report that three Lactobacillus spp. could not withstand $0.5 \%$ phenol (Divisekera et al., 2019).

A major limitation of this study is that we did not simulate the biological nor the physical conditions such as microbiome and lack of oxygen. Additionally, most factors affecting survival were evaluated under the standard conditions and not in the simulated GI environment; furthermore, visual and microscopic analyses were not conducted. Thus, further studies should aim to evaluate the survivability of the $K$. marxianus strains in vivo and microscopical analysis is needed to support the findings described in the present study.

\section{CONCLUSION}

The survivability/growth potential of $K$. marxianus strains was greater than that of Sb MYA-796 under simulated GI conditions. This could be attributed to the extensive spectrum of biochemical activities of $K$. marxianus strains. It was also assumed that higher hydrophobicity, biofilm-forming and auto-aggregation abilities, as well as phenol tolerance in $K$. marxianus strains than Sb MYA796 , could strongly correlate with the superior survivability of $K$. marxianus. In conclusion, our study will provide a basis for understanding the correlations among the survivability and other characteristics the newly isolated $K$. marxianus strains from kefir.

\section{DATA AVAILABILITY STATEMENT}

The original contributions presented in the study are included in the article/Supplementary Material, further inquiries can be directed to the corresponding author.

\section{AUTHOR CONTRIBUTIONS}

H-YY: conceptualization, methodology, investigation, formal analysis, data curation, and writing-original draft. D-HK: conceptualization, methodology, data curation, isolation of Kluyveromyces marxianus strains, and writing-original draft. $\mathrm{H}-\mathrm{JK}$ : investigation, formal analysis, and writing-original draft. 
DB and K-YS: writing-review and editing. HK: supervision, funding acquistion, and writing-review and editing. K-HS: supervision, funding acquisition, writing - review and editing, and project administration. All authors contributed to the article and approved the submitted version.

\section{FUNDING}

This work was supported by the Korea Institute of Planning and Evaluation for Technology in Food, Agriculture, Forestry (IPET) through the Useful Agricultural Life Resources Industry Technology Development Program, funded by the

\section{REFERENCES}

Abdulla, A. A., Abed, T. A., and Saeed, A. M. (2014). Adhesion, autoaggregation and hydrophobicity of six Lactobacillus strains. Microbiol. Res. Int. 4, 381-391. doi: $10.9734 /$ bmrj/2014/6462

Adam, A. C., Rubio-Texeira, M., and Polaina, J. (2005). Lactose: the milk sugar from a biotechnological perspective. Crit. Rev. Food Sci. Nutr. 44, 553-557. doi: 10.1080/10408690490931411

Arioli, S., Ragg, E., Scaglioni, L., Fessas, D., Signorelli, M., Karp, M., et al. (2010). Alkalizing reactions streamline cellular metabolism in acidogenic microorganisms. PLoS One 5:e15520. doi: 10.1371/journal.pone.0015520

Bae, D., Kim, D. H., Chon, J. W., Song, K. Y., and Seo, K. H. (2020). Synergistic effects of the early administration of Lactobacillus kefiranofaciens DN1 and Kluyveromyces marxianus KU140723-05 on the inhibition of Salmonella enteritidis colonization in young chickens. Poul. Sci. 99, 5999-6006. doi: 10. 1016/j.psj.2020.07.032

Bao, Y., Zhang, Y., Zhang, Y., Liu, Y., Wang, S., Dong, X., et al. (2010). Screening of potential probiotic properties of Lactobacillus fermentum isolated from traditional dairy products. Food Control 21, 695-701. doi: 10.1016/j.foodcont. 2009.10.010

Bellon-Fontaine, M. N., Rault, J., and Van Oss, C. J. (1996). Microbial adhesion to solvents: a novel method to determine the electron-donor/electron-acceptor or Lewis acid-base properties of microbial cells. Colloids Surf. B. 7, 47-53. doi: 10.1016/0927-7765(96)01272-6

Ceugniez, A., Coucheney, F., Jacques, P., Daube, G., Delcenserie, V., and Drider, D. (2017). Anti-Salmonella activity and probiotic trends of Kluyveromyces marxianus S-2-05 and Kluyveromyces lactis S-3-05 isolated from a French cheese, Tomme d'Orchies. Res. Microbiol. 168, 575-582. doi: 10.1016/j.resmic. 2017.03.004

Cudennec, B., Balti, R., Ravallec, R., Caron, J., Bougatef, A., Dhulster, P., et al. (2015). In vitro evidence for gut hormone stimulation release and dipeptidylpeptidase IV inhibitory activity of protein hydrolysate obtained from cuttlefish (Sepia officinalis) viscera. Food Res. Int. 78, 238-245. doi: 10.1016/j.foodres. 2015.10.003

Cuperus, P. L., van der Mei, H. C., Reid, G., Bruce, A. W., Khoury, A. H., Rouxhet, P. G., et al. (1993). Physicochemical surface characteristics of urogenital and poultry lactobacilli. J. Colloid Interface Sci. 156, 319-324. doi: 10.1006/jcis. 1993. 1118

Czerucka, D., Piche, T., and Rampal, P. (2007). Review article: yeast as probioticsSaccharomyces boulardii. Aliment. Pharmacol. Ther. 26, 767-778. doi: 10.1111/ j.1365-2036.2007.03442.x

Dertli, E., Mayer, M. J., and Narbad, A. (2015). Impact of the exopolysaccharide layer on biofilms, adhesion and resistance to stress in Lactobacillus johnsonii FI9785. BMC Microbiol. 15:8. doi: 10.1186/s12866-015-0347-2

Diosma, G., Romanin, D. E., Rey-Burusco, M. F., Londero, A., and Garrote, G. L. (2014). Yeasts from kefir grains: isolation, identification, and probiotic characterization. World J. Microbiol. Biotechnol. 30, 43-53. doi: 10.1007/ s11274-013-1419-9

Divisekera, D. M. W. D., Samarasekera, J. K. R. R., Hettiarachchi, C., Gooneratne, J., Choudhary, M. I., Gopalakrishnan, S., et al. (2019). Lactic acid bacteria isolated from fermented flour of finger millet, its probiotic attributes and
Ministry of Agriculture, Food and Rural Affairs (MAFRA, 121051022HD020). This research was also supported by a grant from the National Research Foundation of Korea (NRF), funded by the Korean government (MSIP, grant number 2021R1A2C2006817).

\section{SUPPLEMENTARY MATERIAL}

The Supplementary Material for this article can be found online at: https://www.frontiersin.org/articles/10.3389/fmicb. 2022.842097/full\#supplementary-material

bioactive properties. Ann. Microbiol. 69, 79-92. doi: 10.1007/s13213-018-1399$\mathrm{y}$

Fadda, M. E., Mossa, V., Deplano, M., Pisano, M. B., and Cosentino, S. (2017). In vitro screening of Kluyveromyces strains isolated from Fiore Sardo cheese for potential use as probiotics. $L W T 75,100-106$.

Ferrero, R. L., Hazell, S. L., and Lee, A. (1988). The urease enzymes of Campylobacter pylori and a related bacterium. J. Med. Microbiol. 27, 33-40. doi: 10.1099/00222615-27-1-33

Fonseca, G. G., Heinzle, E., Wittmann, C., and Gombert, A. K. (2008). The yeast Kluyveromyces marxianus and its biotechnological potential. Appl. Microbiol. Biotechnol. 79, 339-354. doi: 10.1007/s00253-008-1458-6

Fonseca, H. C., de Sousa Melo, D., Ramos, C. L., Dias, D. R., and Schwan, R. F. (2021). Probiotic properties of Lactobacilli and their ability to inhibit the adhesion of enteropathogenic bacteria to Caco-2 and HT-29 Cells. Probiotics Antimicrob. Prot. 13, 102-112. doi: 10.1007/s12602-020-09659-2

Hughes, R., Magee, E. A. M., and Bingham, S. (2000). Protein degradation in the large intestine: relevance to colorectal cancer. Curr. Issues Intest. Microbiol. 1, 51-58.

Jeong, D., Kim, D. H., Song, K. Y., and Seo, K. H. (2018). Antimicrobial and antibiofilm activities of Lactobacillus kefiranofaciens DD2 against oral pathogens. J. Oral Microbiol. 10:1472985. doi: 10.1080/20002297.2018.1472985

Kelesidis, T., and Pothoulakis, C. (2012). Efficacy and safety of the probiotic Saccharomyces boulardii for the prevention and therapy of gastrointestinal disorders. Ther. Adv. Gastroenterol. 5, 111-125. doi: $10.1177 / 1756283 \times 11428502$

Kim, D. H., Chon, J. W., Kim, H., Kim, H. S., Choi, D., Hwang, D. G., et al. (2015). Detection and enumeration of lactic acid bacteria, acetic acid bacteria and yeast in kefir grain and milk using quantitative real-time PCR. J. Food Safety 35, 102-107. doi: 10.1111/jfs. 12153

Kim, D. H., Jeong, D., Kang, I. B., Kim, H., Song, K. Y., and Seo, K. H. (2017). Dual function of Lactobacillus kefiri DH5 in preventing high-fat-dietinduced obesity: direct reduction of cholesterol and upregulation of PPAR- $\alpha$ in adipose tissue. Mol. Nutr. Food Res. 61:700252. doi: 10.1002/mnfr.20170 0252

Kim, D. H., Jeong, D., Kang, I. B., Lim, H. W., Cho, Y., and Seo, K. H. (2019a). Modulation of the intestinal microbiota of dogs by Kefir as a functional dairy product. J. Dairy Sci. 102, 3903-3911. doi: 10.3168/jds.201815639

Kim, D. H., Jeong, D., Kim, H., and Seo, K. H. (2019b). Modern perspectives on the health benefits of Kefir in next generation sequencing era: improvement of the host gut microbiota. Crit. Rev. Food Sci. Nutr. 59, 1782-1793. doi: 10.1080/ 10408398.2018.1428168

Kim, D. H., Kim, H., and Seo, K. H. (2020). Microbial composition of Korean kefir and antimicrobial activity of Acetobacter fabarum DH1801. J. Food Safety 40:e12728. doi: 10.1111/jfs. 12728

Kos, B. V. Z. E., Šušković, J., Vuković, S., Šimpraga, M., Frece, J., and Matošić, S. (2003). Adhesion and aggregation ability of probiotic strain Lactobacillus acidophilus M92. J. Appl. Microbiol. 94, 981-987.

Lane, M. M., and Morrissey, J. P. (2010). Kluyveromyces marxianus: a yeast emerging from its sister's shadow. Fungal Biol. Rev. 24, 17-26. doi: 10.1016/j. fbr.2010.01.001 
Lim, H. W., Kim, D. H., Jeong, D., Kang, I. B., Kim, H., and Seo, K. H. (2019). Biochemical characteristics, virulence traits and antifungal resistance of two major yeast species isolated from Kefir: kluyveromyces marxianus and Saccharomyces unisporus. Int. J. Dairy Technol. 72, 275-281. doi: 10.1111/14710307.12582

Liu, H., Xie, Y. H., Xiong, L. X., Dong, R. T., Pan, C. L., Teng, G. X., et al. (2012). Effect and mechanism of cholesterol-lowering by Kluyveromyces from Tibetan kefir. Adv. Mater. Res. 343, 1290-1298. doi: 10.4028/www.scientific.net/AMR. 343-344.1290

LoCascio, R. G., Desai, P., Sela, D. A., Weimer, B., and Mills, D. A. (2010). Broad conservation of milk utilization genes in Bifidobacterium longum subsp. infantis as revealed by comparative genomic hybridization. Appl. Environ. Microbiol. 76, 7373-7381. doi: 10.1128/AEM.00675-10

Mladenović, K. G., Grujović, M. Ž, Nikodijević, D., and ČomiĆ, L. R. (2020). The hydrophobicity of enterobacteria and their co-aggregation with Enterococcus faecalis isolated from Serbian cheese. Biosci. Microbiota. Food Health 39, $227-$ 233.

Mora, D., Maguin, E., Masiero, M., Parini, C., Ricci, G., Manachini, P. L., et al. (2004). Characterization of urease genes cluster of Streptococcus thermophilus. J. Appl. Microbiol. 96, 209-219. doi: 10.1046/j.1365-2672.2003. 02148.x

Moradi, R., Nosrati, R., Zare, H., Tahmasebi, T., Saderi, H., and Owlia, P. (2018). Screening and characterization of in-vitro probiotic criteria of Saccharomyces and Kluyveromyces strains. Iran J. Microbiol. 10:123.

Nwanyanwu, C. E., Alisi, C. S., Nweke, C. O., and Orji, J. C. (2012). Cell surface properties of phenol-utilizing bacteria isolated from petroleum refinery wastewater. J. Res. Biol. 2, 383-391.

Organic Materials Review Institute [OMRI] (2014). Yeast. 1-29. Available online at: https://www.ams.usda.gov/sites/default/files/media/Yeast\%20TR\% 20Handling\%201-22-14\%20final.pdf (accessed on Nov 16, 2021)

Pan'kova, N. V., Polyudova, T. V., Lemkina, L. M., and Korobov, V. P. (2011). Hydrophobic and donor-acceptor properties of the surface of warnerinsensitive or-resistant staphylococcus cells. Microbiology 80, 573-575. doi: 10. 1134/S0026261711040163

Quigley, E. M. (2019). Prebiotics and probiotics in digestive health. Clin. Gastroenterol. Hepatol. 17, 333-344. doi: 10.1016/j.cgh.2018.09.028

Rabot, S., Rafter, J., Rijkers, G. T., Watzl, B., and Antoine, J. M. (2010). Guidance for substantiating the evidence for beneficial effects of probiotics: impact of probiotics on digestive system metabolism. J. Nutr. 140, 677S-689S. doi: 10. 3945/jn.109.113738

Romanin, D. E., Llopis, S., Genovés, S., Martorell, P., Ramón, V. D., Garrote, G. L., et al. (2016). Probiotic yeast Kluyveromyces marxianus CIDCA 8154 shows anti-inflammatory and anti-oxidative stress properties in in vivo models. Benef. Microbes 7, 83-93. doi: 10.3920/BM2015.0066

Rowland, I., Gibson, G., Heinken, A., Scott, K., Swann, J., Thiele, I., et al. (2018). Gut microbiota functions: metabolism of nutrients and other food components. Eur. J. Nutr. 57, 1-24. doi: 10.1007/s00394-017-1445-8

Sánchez, B., Delgado, S., Blanco-Míguez, A., Lourenço, A., Gueimonde, M., and Margolles, A. (2017). Probiotics, gut microbiota, and their influence on host health and disease. Mol. Nutr. Food Res. 61:1600240. doi: 10.1002/mnfr. 201600240

Sazawal, S., Hiremath, G., Dhingra, U., Malik, P., Deb, S., and Black, R. E. (2006). Efficacy of probiotics in prevention of acute diarrhoea: a meta-analysis of masked, randomised, placebo-controlled trials. Lancet Infect. Dis. 6, 374-382. doi: 10.1016/S1473-3099(06)70495-9

Shehata, M. G., El Sohaimy, S. A., El-Sahn, M. A., and Youssef, M. M. (2016). Screening of isolated potential probiotic lactic acid bacteria for cholesterol lowering property and bile salt hydrolase activity. Ann. Agric. Sci. 61, 65-75. doi: 10.1016/j.aoas.2016.03.001

Simova, E., Beshkova, D., Angelov, A., Hristozova, T., Frengova, G., and Spasov, Z. (2002). Lactic acid bacteria and yeasts in kefir grains and Kefir made from them. J. Ind. Microbiol. Biotechnol. 28, 1-6. doi: 10.1038/sj.jim.700 0186

Song, M., Yun, B., Moon, J. H., Park, D. J., Lim, K., and Oh, S. (2015). Characterization of selected Lactobacillus strains for use as probiotics. Korean J. Food Sci. Anim. Resour. 35, 551-556. doi: 10.5851/kosfa.2015.35. 4.551

Suvarna, S., Dsouza, J., Ragavan, M. L., and Das, N. (2018). Potential probiotic characterization and effect of encapsulation of probiotic yeast strains on survival in simulated gastrointestinal tract condition. Food Sci. Biotechnol. 27, 745-753. doi: 10.1007/s10068-018-0310-8

Tambekar, D. H., and Bhutada, S. A. (2010). An evaluation of probiotic potential of Lactobacillus sp. From milk of domestic animals and commercial available probiotic preparations in prevention of enteric bacterial infections. Recent Res. Sci. Technol. 2, 82-88.

Tzounis, X., Vulevic, J., Kuhnle, G. G., George, T., Leonczak, J., Gibson, G. R., et al. (2008). Flavanol monomer-induced changes to the human faecal microflora. $\mathrm{Br}$. J. Nutr. 99, 782-792. doi: 10.1017/S0007114507853384

Xanthopoulos, V., Litopoulou-Tzanetaki, E., and Tzanetakis, N. (2000). Characterization of Lactobacillus isolates from infant faeces as dietary adjuncts. Food Microbiol. 17, 205-215.

You, S. J., Cho, J. K., Ha, C. G., Kim, C. H., and Heo, K. C. (2006). Probiotic properties of the Candida kefyr isolated from kefir. J. Anim. Sci. Technol. 48, 307-314. doi: 10.5187/jast.2006.48.2.307

Youn, H. Y., Kim, D. H., Kim, H. J., Jang, Y. S., Song, K. Y., Bae, D., et al. (2022). A Combined In Vitro and In Vivo Assessment of the Safety of the Yeast Strains Kluyveromyces marxianus A4 and A5 Isolated from Korean Kefir. Probiotics Antimicrob. Proteins [Epub online ahead of print] doi: 10.1007/s12602-02109872-7

Conflict of Interest: The authors declare that the research was conducted in the absence of any commercial or financial relationships that could be construed as a potential conflict of interest.

Publisher's Note: All claims expressed in this article are solely those of the authors and do not necessarily represent those of their affiliated organizations, or those of the publisher, the editors and the reviewers. Any product that may be evaluated in this article, or claim that may be made by its manufacturer, is not guaranteed or endorsed by the publisher.

Copyright (c) 2022 Youn, Kim, Kim, Bae, Song, Kim and Seo. This is an open-access article distributed under the terms of the Creative Commons Attribution License (CC BY). The use, distribution or reproduction in other forums is permitted, provided the original author(s) and the copyright owner(s) are credited and that the original publication in this journal is cited, in accordance with accepted academic practice. No use, distribution or reproduction is permitted which does not comply with these terms. 